\title{
Broadening Access to Space Science Data: The Astromaterials Data System
}

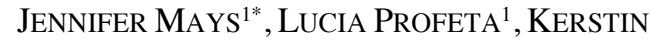 \\ LEHNERT $^{1}$, PENG J I ${ }^{1}$, SHAUNNA MORRISON ${ }^{2}$, ANNIKA \\ JOHANSSON $^{1}$, LULIN SONG ${ }^{1}$, JUAN DAVID FIGUEROA ${ }^{1}$, \\ CINDY EVANS ${ }^{3}$, RYAN ZEIGLER ${ }^{3}$ \\ ${ }^{1}$ Lamont-Doherty Earth Observatory, Columbia University, \\ Palisades, NY, USA (*correspondence: \\ jmays@1deo.columbia.edu) \\ ${ }^{2}$ Carnegie Science Earth and Planets, Washington, DC, USA \\ ${ }^{3}$ NASA Johnson Space Center, Houston, TX, USA
}

The Astromaterials Data System (AstroMat) is being developed as a comprehensive data system for all analytical data generated from samples hosted at NASA's Johnson Space Center (JSC) Astromaterials Acquisition and Curation Office.

The goal of Astromat is to be an integrated resource for persistent online access, archiving, and exploration of lunar and planetary analytical data.

As a first step, Astromat has compiled a bibliography of over 1700 references that contain data for specimens in all JSC sample collections. Data from more than 800 references pertaining to samples from the Apollo Missions and lunar meteorites were already compiled as part of the MoonDB project and have been migrated to the new AstroDB.

Efforts currently focus on the development of user interfaces for searching and accessing and on ingestion of meteorite data.

Meteorite metadata profiles will be informed by the metadata contained in the Meteoritical Bulletin Database and will be made accessible to the community for comments and review.

We encourage the community to help continue to advise and inform on the usability and functionality of the interfaces in order for AstroMat to meet its goals of furthering planetary science research. 